\title{
KBMA Listeria monocytogenes is an effective vector for DC-mediated induction of antitumor immunity
}

\author{
Mojca Skoberne, ${ }^{1}$ Alice Yewdall, ${ }^{1}$ Keith S. Bahjat, ${ }^{2}$ Emmanuelle Godefroy, ${ }^{1}$ \\ Peter Lauer, ${ }^{2}$ Edward Lemmens, ${ }^{2}$ Weiqun Liu, ${ }^{2}$ Will Luckett, ${ }^{2}$ Meredith Leong, ${ }^{2}$ \\ Thomas W. Dubensky, ${ }^{2}$ Dirk G. Brockstedt, ${ }^{2}$ and Nina Bhardwaj ${ }^{1}$ \\ ${ }^{1}$ Cancer Institute, New York University School of Medicine, New York, New York, USA. ${ }^{2}$ Anza Therapeutics, Concord, California, USA.
}

\begin{abstract}
Vaccine strategies that utilize human DCs to enhance antitumor immunity have yet to realize their full potential. Approaches that optimally target a spectrum of antigens to DCs are urgently needed. Here we report the development of a platform for loading DCs with antigen. It is based on killed but metabolically active (KBMA) recombinant Listeria monocytogenes and facilitates both antigen delivery and maturation of human DCs. Highly attenuated KBMA L. monocytogenes were engineered to express an epitope of the melanoma-associated antigen MelanA/Mart-1 that is recognized by human $\mathrm{CD8}^{+} \mathrm{T}$ cells when presented by the MHC class I molecule HLA$A^{*} 0201$. The engineered KBMA $L$. monocytogenes induced human DC upregulation of costimulatory molecules and secretion of pro-Th1 cytokines and type I interferons, leading to effective priming of Mart-1-specific human $\mathrm{CD8}^{+} \mathrm{T}$ cells and lysis of patient-derived melanoma cells. KBMA L. monocytogenes expressing full-length NY-ESO-1 protein, another melanoma-associated antigen, delivered the antigen for presentation by MHC class I and class II molecules independent of the MHC haplotype of the DC donor. A mouse therapeutic tumor model was used to show that KBMA L. monocytogenes efficiently targeted APCs in vivo to induce protective antitumor responses. Together, our data demonstrate that KBMA L. monocytogenes may be a powerful platform that can both deliver recombinant antigen to DCs for presentation and provide a potent DC-maturation stimulus, making it a potential cancer vaccine candidate.
\end{abstract}

\section{Introduction}

Melanoma is one of the most rapidly growing cancers worldwide, yet there is no satisfactory treatment either in the adjuvant setting or when it has advanced to metastatic disease. Several approaches to boost antitumor immune responses in melanoma have been attempted (1). While a few of these have successfully elicited high frequencies of melanoma-associated antigen-reactive T cells (2-4), their induction does not correlate with tumor regression. Complex and tailored therapies (e.g., adoptive T cell therapy or administration of anti-CTLA antibodies), have shown some success in reducing high tumor burden (5-7), albeit with high-grade toxicity. Therefore, alternative approaches are needed.

DCs are attractive cellular adjuvants that can increase host resistance to tumors (8). To optimally prime naive T cells, DCs must not only present high levels of MHC-peptide complexes but must also undergo activation and maturation. An ideal DC-targeting vaccine formulation, therefore, should simultaneously deliver

Nonstandard abbreviations used: Cr-51, Chromium-51; ELISPOT, enzyme-linked immunospot; HK, heat killed; Inl, internalin; KBMA, killed but metabolically active; LLO, listeriolysin O; $L m A g$, HLA-A*0201-restricted CD8 ${ }^{+} \mathrm{T}$ cell epitopes of MP and melanoma-associated antigen MelanA/Mart-1 protein; $L m N Y$, L. monocytogenes encoding full-length NY-ESO-1 protein; MC, a cocktail of IL-6, IL-1 $\beta$, TNF- $\alpha$, and PGE ; $_{2}$ moDC, monocyte-derived DC; MP, influenza matrix protein; S-59, synthetic psoralen DNA cross-linking agent; UVA, long-wave UV light.

Conflict of interest: T.W. Dubensky Jr., D.G. Brockstedt, K.S. Bahjat, P. Lauer, M Leong, W. Luckett, W. Liu, and E. Lemmens are or were employees of Anza Therapeutics, which owns intellectual property covering the compositions and methods described in this manuscript. N. Bhardwaj was a former Science Advisory Board (SAB) member of Anza Therapeutics at the time of the study design. In addition, Anza Therapeutics employees hold stock options in the company. N. Bhardwaj is a coinventor of patents pertaining to human dendritic cell preparation.

Citation for this article: J. Clin. Invest. doi:10.1172/JCI31350. antigens and activate DCs to enhance expression of surface MHCpeptide complexes and costimulatory molecules and production of cytokines that prime potent $\mathrm{CD}^{+}$and $\mathrm{CD} 4^{+} \mathrm{T}$ cell responses. To date, there is no standard, efficient, and cost-effective method that combines antigen loading and activation/maturation of DCs for clinical immunotherapy.

Listeria monocytogenes are Gram-positive facultative intracellular bacteria that are internalized by phagocytic and nonphagocytic cells. Previous studies have shown that self or heterologous antigens encoded by $L$. monocytogenes or the recombinant $L$. monocytogenes strains can access endogenous and exogenous antigen processing and presentation pathways to prime $\mathrm{CD}^{+}$and $\mathrm{CD}^{+} \mathrm{T}$ cells, respectively. Following internalization, L. monocytogenes gain access to the cytosol of APCs from the phagosome through expression of listeriolysin $\mathrm{O}$ (LLO). In addition, despite its preferential intracytoplasmic location, $L$. monocytogenes very efficiently prime $\mathrm{CD}^{+} \mathrm{T}$ cell responses (9). These characteristics have made $L$. monocytogenes an attractive and efficacious platform in murine models of cancer and microbial infection. In humans, infection with live WT or recombinant L. monocytogenes activates DCs in vitro (10); however, for broad clinical application one must address pathogenicity while preserving immunogenicity of recombinant $L$. monocytogenes vaccines.

The potency of killed but metabolically active (KBMA) recombinant $L$. monocytogenes was recently demonstrated in an animal model of tumor immunity (11). KBMA L. monocytogenes are rendered noninfectious by the combined use of psoralen and longwave UV light (UVA) and abrogation of nucleotide excision repair through deletion of the bacterial uvrAB genes. While KBMA L. monocytogenes cannot propagate, they remain metabolically active and express their genetic repertoire, including the heterologous 
antigens they were engineered to express (11). Most importantly, KBMA L. monocytogenes induced cytolytic $\mathrm{CD}^{+} \mathrm{T}$ cell responses to an endogenous tumor epitope and reduced tumor burden in a therapeutic CT-26 tumor model (10).

Several murine studies have provided us with evidence of the fundamental relationship between $L$. monocytogenes and endogenous DCs during an infection. Priming of naive $\mathrm{CD}^{+} \mathrm{T}$ cells to L. monocytogenes-specific antigens is mediated by DCs (12). Contrary to previous assumptions, DCs are also required to maximally activate a recall response to various pathogens, including L. monocytogenes (13). These 2 events may be related to the fact that DCs are necessary for the efficient entry of L. monocytogenes into the spleen, in which $\mathrm{T}$ cell activation takes place (14). All together, this makes L. monocytogenes a promising candidate for development of human DC-targeting vaccines.

We report here that KBMA recombinant $L$. monocytogenes can be used as an antigen-loading vector to target human DCs. KBMA L. monocytogenes encoding tumor antigens induce DC maturation, secretion of Th1-polarizing cytokines and type I interferons, and expression of the recombinant tumor antigen and confer potent antigen priming activity to these APCs. Once infected with KBMA L. monocytogenes expressing full-length NY-ESO-1 protein, DCs presented antigens in the context of both class I and class II MHC molecules by donors with different MHC haplotypes. Furthermore, antigen-primed $\mathrm{CD} 8^{+} \mathrm{T}$ cells acquired effector function and lysed a patient-derived melanoma tumor cell line. Thus, recombinant $L$. monocytogenes are a unique antigen delivery vehicle that can mature DCs for T cell priming, while simultaneously delivering both $\mathrm{CD}^{+}$and $\mathrm{CD}^{+} \mathrm{T}$ cell-restricted epitopes for presentation, independently of patient's MHC restriction.

\section{Results}

Deletion of selected virulence proteins preserves the DC activation potential of $L$. monocytogenes. The ability of $L$. monocytogenes to infect phagocytic cells, as well as to access the host cell cytosol following the escape from the phagolysosome, renders these bacteria a suitable vaccine platform for the delivery of antigens into the endogenous and exogenous pathways of antigen processing by DCs. It has been reported that WT L. monocytogenes (i.e., fully infectious and replicating L. monocytogenes) are extremely efficient at activating human monocyte-derived DCs (moDCs) (10). Their use, however, is contraindicated in humans. Therefore, we first tested whether highly attenuated strains of $L$. monocytogenes, which are suitable for clinical applications, retain their ability to efficiently activate DCs in vitro. The bacterial genes act $A$, inlB, and bly encode 3 important $L$. monocytogenes virulence determinants: ActA, which mediates actin polymerization; internalin B (inlB), which facilitates cell binding and invasion; and pore-forming LLO, which permits escape from phagolysosomes to cytosol. L. monocytogenes strains attenuated by deletion of either both the act $A$ and inlB genes ( $\triangle a c t A \Delta i n l B L$. monocytogenes) or the bly gene ( $\triangle$ LLO L. monocytogenes) were compared with WT L. monocytogenes in their ability to stimulate DCs to upregulate maturation markers, secrete proinflammatory cytokines, and stimulate allogeneic $\mathrm{T}$ cells. As anticipated, presence of LLO enhanced activation of DCs (Figure 1). Confirming previously reported data (15), deletion of the bly gene, to some extent, impaired the ability of DCs to upregulate the costimulatory molecules CD80 and CD83 (Figure 1, A and B) as well as the proinflammatory and pro-Th1 cytokines IL-1 $\beta$ (data not shown), TNF- $\alpha$, and IL-12p70 (Figure 1C). However, the difference was only observed at lower MOI. Activation of DCs with $\triangle a c t A \Delta i n l B$
L. monocytogenes did not statistically significantly impair DC maturation when compared with the parent strain (Supplemental Table 1). In accordance with these findings, DCs infected with any of the attenuated strains induced proliferation of allogeneic T cells comparable to control DCs matured with a cocktail of inflammatory cytokines (a combination of IL-6, IL-1 $\beta$, TNF- $\alpha$, and PGE 2 hereafter referred to as MC) (Figure 1D). Thus, the ability to induce DC maturation was not impaired with the live-attenuated $L$. monocytogenes strain deleted of 2 virulence factors ActA and InlB.

KBMA L. monocytogenes are stimulatory for human DCs. While heatkilled (HK) vaccines are safer to use, this is often at the expense of their immunogenicity. To further improve safety, we engineered a KBMA $L$. monocytogenes vaccine candidate on the $\triangle a c t A \Delta i n l B \Delta w r A B$ L. monocytogenes background as described previously (11). KBMA L. monocytogenes could no longer replicate, but they maintained metabolic activity, retained the ability to exit the phagolysosome, and escaped into the cytosol only where they colocalized with actin (Figure 2A). Therefore, recombinant antigen-expressing KBMA L. monocytogenes, in contrast to killed L. monocytogenes, have the potential to deliver antigens to the endogenous antigen processing pathway, a necessity for a successful vaccine vector, without the associated risks of a replicating live bacterium. The KBMA L. monocytogenes strain was compared with live, killed, and metabolically nonactive $L$. monocytogenes (inactivated with a high synthetic psoralen DNA cross-linking agent [S-59] concentration) with the same genetic background (Figure 2 and Supplemental Figure 1). The ability of KBMA L. monocytogenes to activate DCs was compared with well-described maturation stimuli LPS, poly I:C; or MC. Live $\triangle a c t A \Delta i n l B \Delta u v r A B$ L. monocytogenes were as effective as 2 positive controls (LPS and MC) in terms of activating DCs (upregulation of typical maturation markers; Figure 2, B and C). Furthermore, the KBMA L. monocytogenes vaccine strain induced maturation of DCs to a similar extent as live bacteria, provided a higher MOI was used (10 vs. 1 MOI) (Figure 2, B and C). A similar pattern was observed with cytokines secreted by DCs upon infection (Figure 2, D-F). Live, KBMA L. monocytogenes, and LPS induced TNF- $\alpha$, IL- 6 , and IL-12p70 to comparable levels and live and KBMA L. monocytogenes induced these to higher levels than HK L. monocytogenes (Figure 2D). Similar results were obtained with CD $11 c^{+}$DCs isolated from blood of healthy donors (Supplemental Figure 2). KBMA and live L. monocytogenes were equivalent inducers of MCP-3 and RANTES, 2 chemokines that recruit $\mathrm{T}$ cells (Figure 2E).

WT L. monocytogenes but not HK $L$. monocytogenes induce type I IFN transcription and secretion in mouse DCs (16). Similarly, secretion of type I IFN- $\alpha$ has also been detected following infection of murine macrophages with WT but not $\Delta$ bly L. monocytogenes strains (17). As shown in Figure 2F, we found that substantial amounts of IFN- $\alpha$ were produced by human moDCs upon exposure to live and KBMA L. monocytogenes but not HK or $\Delta$ bly L. monocytogenes, confirming the requirement for cytoplasmic entry of bacteria.

CCR7 is a chemokine receptor that recognizes the chemokines CCL19 and CCL21 produced in lymphatic vessel endothelial cells and $\mathrm{T}$ cell areas of lymph nodes and is therefore essential for trafficking of DCs to lymph nodes. Flow cytometry analysis revealed that infection with live and KBMA L. monocytogenes induced expression of CCR7 on DCs (Figure 2, G and H). The onset of CCR7 expression was rapid, commencing 6 hours after infection, with high levels maintained 24 hours after infection. The levels and kinetics of expression of CCR7 were comparable among LPSmatured DCs or DCs infected with live or KBMA L. monocytogenes 

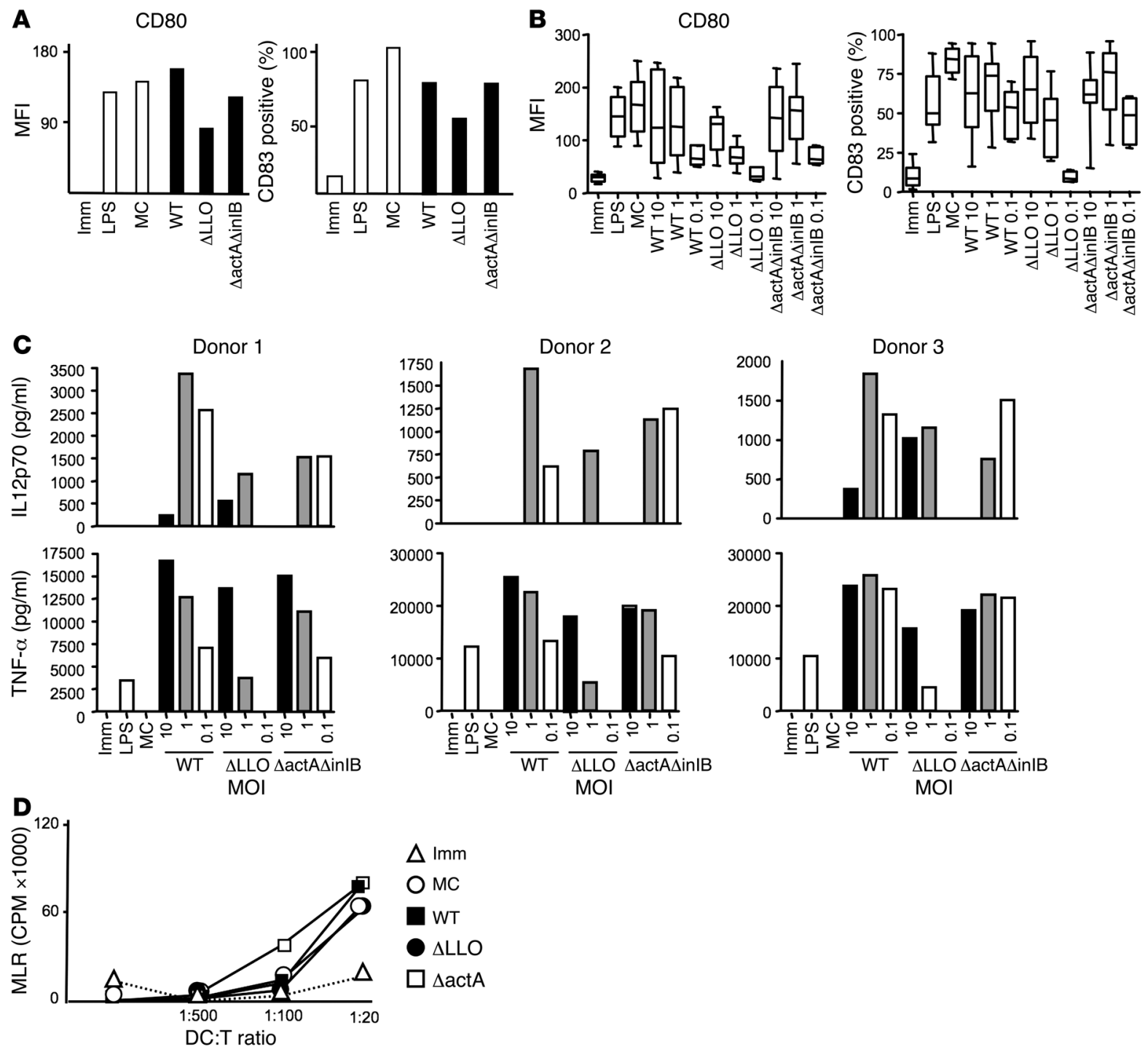

Figure 1

Deletion of virulence factors and immunogenicity of $L$. monocytogenes. Live-attenuated L. monocytogenes strains $(L m)$ deleted of either $h l y$ $(\Delta \mathrm{LLO})$ or actA and inIB ( $\triangle$ actA $\Delta$ inIB) were compared with WT L. monocytogenes, LPS, unstimulated (Imm), or a cocktail of inflammatory cytokines (MC) in their potency to mature human moDCs. (A and B) DCs were harvested 40 hours after infection with $L$. monocytogenes (MOI 1), and expression of CD80 and CD83 was assessed by flow cytometry. (A) Data for a single donor (B) and median value (horizontal line) and range (vertical line) with 25th and 75th percentiles (bars) of at least 7 individual results are shown. (C) At the same time supernatants from control and L. monocytogenes-infected DC cultures were collected (white bars, MOI 0.1 or controls; gray bars, MOI 1; black bars, MOI 10) for quantitation of IL-12p70 and TNF- $\alpha$ by cytokine bead array assay. Results of 3 representative donors are shown. (D) DCs were generated as above and cultured with allogeneic naive CD4 ${ }^{+} \mathrm{T}$ cells for 4 days. T cell proliferation was measured by incorporation of radioactive thymidine during the last 16 hours. A representative experiment of 3 is shown.

strains and were superior to HK L. monocytogenes-infected DCs. Altogether, our data indicate that the coincubation of the KBMA L. monocytogenes vaccine strain with human DCs leads to full maturation of DCs and optimally equips them for priming.

KBMA L. monocytogenes encoding recombinant antigens access the endogenous pathway and stimulate antigen-specific $C D 8^{+} T$ cells. To test the ability of KBMA bacteria to load antigen into the MHC class I pathway of APCs, $\triangle a c t A \triangle i n l B \triangle w v r A B$ L. monocytogenes were constructed to express HLA-A*0201-restricted CD8 ${ }^{+} \mathrm{T}$ cell epitopes of influenza matrix protein (MP) and melanoma-associated antigen MelanA/Mart-1 protein ( $L m A g)$. DCs infected with
KBMA LmAg at MOI 100 were compared with DCs infected with live $L m A g$ (MOI 10) (Figure 3A). DCs infected with empty vector served as negative controls. Forty hours after infection, the DCs were washed and cocultured with $\mathrm{MP}_{58-66^{-}}$or MART $1_{26-35^{-}}$ specific $\mathrm{CD}^{+} \mathrm{T}$ cell clones for 24 hours. The number of cells synthesizing IFN- $\gamma$ was enumerated by enzyme-linked immunospot (ELISPOT) assay. It should be pointed out that in this assay the number of responding $\mathrm{T}$ cell clones depends on multiple factors and not every clone will respond, even when DCs are optimally loaded with antigens (18). Activation of antigen-specific $T$ cell clones was observed with HLA-A*0201+ DCs infected with either 
A

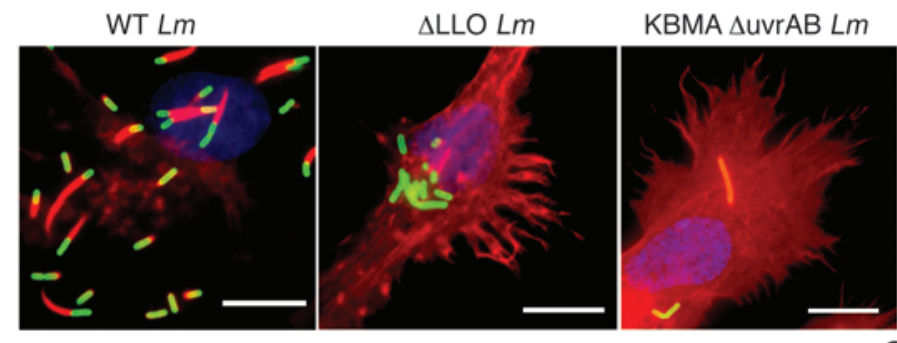

B
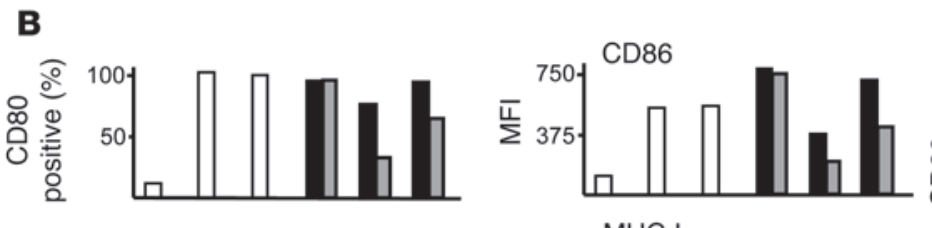

C
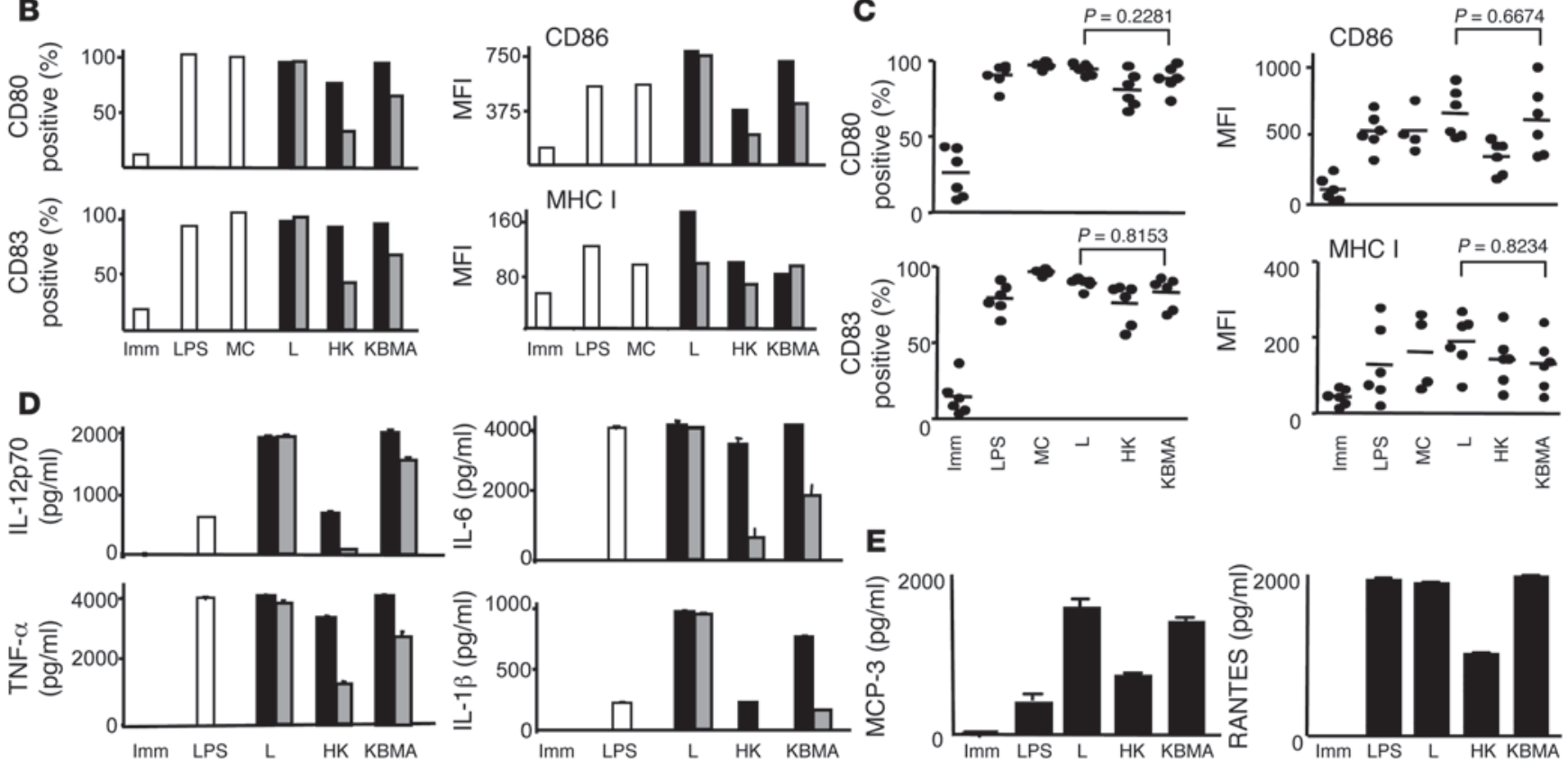

$\mathbf{F}$
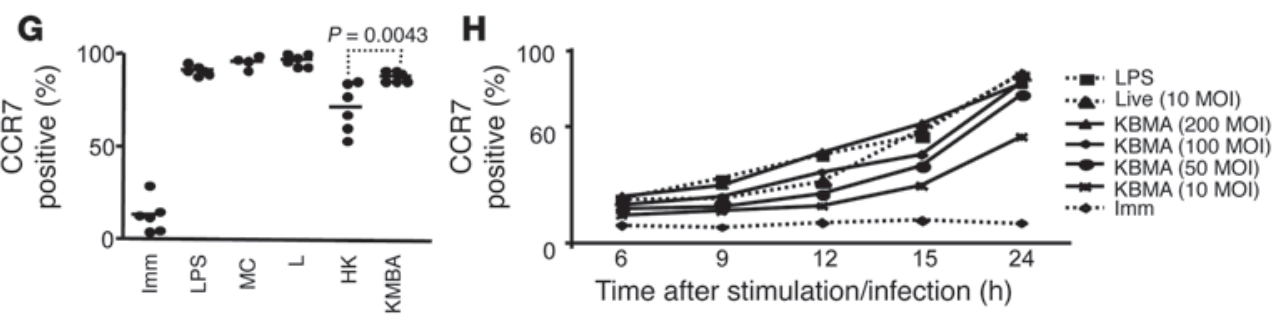

Figure 2

KBMA L. monocytogenes activate moDCs. (A) DCs were infected with L. monocytogenes strains, stained with antibodies to $L$. monocytogenes (green), phalloidin (red), and DAPI (blue). Visualization of polymerized actin and colocalization with the bacteria (yellow) confirms the presence of bacteria within the cytoplasm. Scale bar: $10 \mu \mathrm{m}$. (B and C) DCs were infected with live (L), HK, or KBMA L. monocytogenes. Unstimulated or DCs, exposed to LPS or MC, were used as controls. Expression of DC-maturation markers for a typical donor is shown (gray bars, MOI 1; black bars, MOI 10) (B). Results of multiple donors are shown in C (MOI 10). (D and E) Presence of cytokines (D) and chemokines (E) was measured by ELISA (gray bars, MOI 1; black bars, MOI 10). (F) DCs were infected at MOI 10 (WT, L) or MOI 200 ( $\Delta$ LLO, HK, and KBMA L. monocytogenes). Immature, LPS, or poly I:C-stimulated DCs were used as controls. IFN- $\alpha$ in supernatants was measured. (G) DCs were infected with live, HK, or KBMA L. monocytogenes and left untreated or stimulated with LPS or MC. CCR7 was measured by flow cytometry after 40 hours. (H) DCs were infected with live or KBMA L. monocytogenes. CCR7 was measured at indicated time points. Numbers in parentheses indicate MOI used for infection. Nonstimulated or LPS-stimulated DCs were used as controls. In C, F, and G, values from individual donors (dots), mean values (lines), and Student's $t$ test $P$ values are shown. In $\mathbf{A}, \mathbf{B}, \mathbf{D}, \mathbf{E}$, and $\mathbf{H}$, a representative experiment of at least 3 performed is shown. In $\mathbf{D}$ and $\mathbf{E}$, error bars represent SD of triplicate culture wells.

live or KBMA $L m A g$, demonstrating that both live or KBMA L. monocytogenes can deliver antigen into the MHC class I pathway of antigen processing. No responses were observed either when HLA-A* $0201^{+}$donor DCs were infected with the empty vector or when HLA-A*0201- donor DCs were infected with $L m A g$, thus confirming antigen specificity. Replication was not necessary for the presentation of recombinant antigens, since KBMA L. monocytogenes strains were able to induce effector responses from antigen-specific T cells. Both live and KBMA L. monocytogenes induced marked responses from $T$ cells as early as 6 hours after infection. DCs infected with KBMA L. monocytogenes sustained expression over the first 12 hours, while those infected with live L. monocytogenes 

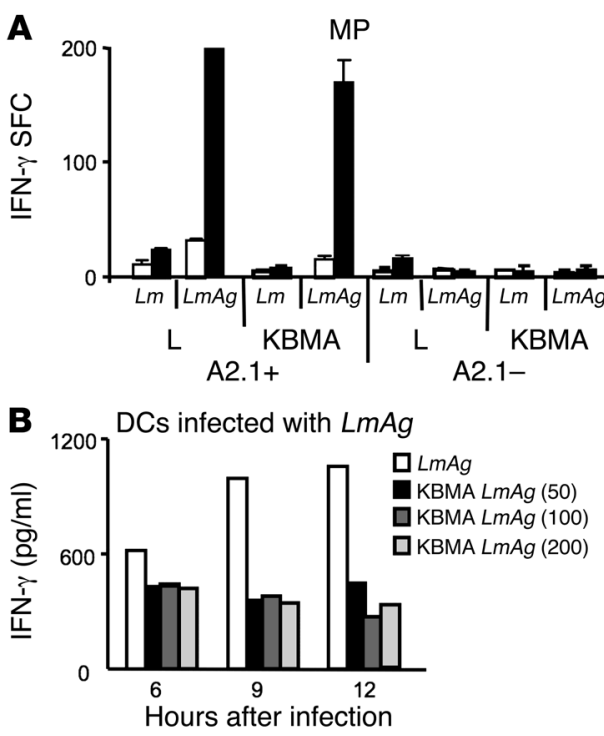

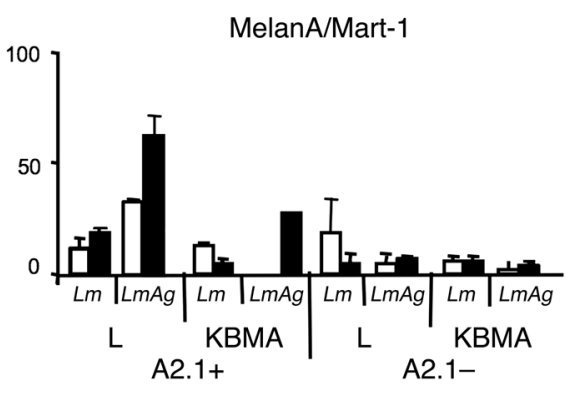

Control samples

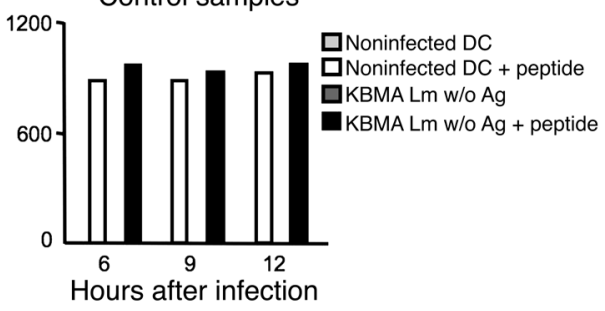

Figure 3

Live and KBMA L. monocytogenes successfully deliver recombinant antigens for presentation by $\mathrm{MHC} \mathrm{I}$ molecules. (A) DCs from HLA A2.1+ or HLA A2.1- donors were infected with recombinant $L$. monocytogenes $\triangle a c t A \Delta i n I B \Delta u v r A B$ strain expressing $L m A g$ or empty vaccine vector (L. monocytogenes) at MOI 10 (live) or MOI 100 (KBMA). After 40 hours, DCs were cocultured with 20,000 MP $_{58-66}$ or Mart- $1_{26-35}$-specific $\mathrm{CD}^{+} \mathrm{T}$ cell clones in an IFN- $\gamma$-capturing ELISPOT assay. The number of spots corresponding to IFN- $\gamma$ secreting T cells (SFC) is shown. Black bars represent responses of T cell-DC cocultures, and white bars represent DC-only cultures. Error bars represent SD of triplicate culture wells. (B) DCs were infected with live or KBMA LmAg (left panel) at the indicated MOI in 96-well plates. As a control, we used noninfected $\mathrm{DCs}$, either unpulsed or pulsed with $\mathrm{MP}_{58-66}$ peptide or DCs infected with $L$. monocytogenes that were either unpulsed or pulsed with $\mathrm{MP}_{58-66}$ (right panel). At indicated times, DCs were fixed and MP-specific T cells were added to the wells. After 18 hours of T cell-DC coculture, supernatants were harvested and the amount of IFN- $\gamma$ produced by T cells was determined by ELISA.

showed an increase in expression over time, likely related to replication of bacteria within the infected cell (Figure 3B). In both groups, expression of heterologous antigen remained detectable as demonstrated by the activation of antigen-specific $\mathrm{T}$ cells up to 40 hours after infection (Figure 3A). This period of antigen expression and recognition would encompass the time required for DCs to prime effector T cells in vivo (19).

KBMA L. monocytogenes encoding full-length NY-ESO-1 protein delivers antigens for presentation by $\mathrm{MHC}$ I and MHC II molecules. Ideally, cancer vaccines should prime both $\mathrm{CD}^{+}$and $\mathrm{CD}^{+} \mathrm{T}$ cells. Immunogenic epitopes have been identified for many cancer-relevant antigens, mostly for HLA A2.1-specific CD8-restricted epitopes, while CD4restricted epitopes have been identified for various $\mathrm{MHC}$ haplotypes $(20,21)$. When individual CD4- and CD8-restricted epitopes are combined into vaccines, only a very small group of patients that receive these treatments have the required combination of $\mathrm{MHC}$ class I and class II molecules to elicit desired responses. Thus, whole proteins are a better alternative, especially when targeted specifically to professional APCs. NY-ESO-1 is a cancer-testis antigen, detectable in up to $30 \%-40 \%$ of metastatic melanomas. It is expressed by a variety of cancers but not in adult somatic tissues, making it an attractive target for immunotherapies (22-24). We have shown previously that when administered with adjuvants, NY-ESO-1 protein induces both humoral and cellular immune responses (25). Here we tested whether KBMA attenuated L. monocytogenes that express the NY-ESO-1 protein are an efficient adjuvant and delivery system.
For the purpose of the experiment, we first generated NY-ESO-1-specific $\mathrm{CD}^{+}$and $\mathrm{CD}^{+} \mathrm{T}$ cell clones. The $\mathrm{CD}^{+}$clones were HLA A2.1 restricted while $\mathrm{CD}^{+}$clones were DR (clone no. 15 , clone no. 18 ) or DQ (clone no. 30) restricted. HLA A2. $1^{+}$or HLA DR $\mathrm{B} 4 * 0101^{+}$DCs were infected either with KBMA $\Delta a c t \Delta i n l B \Delta u v r$ L. monocytogenes encoding full-length NY-ESO-1 protein $(L m N Y)$ or its empty vector control (L. monocytogenes). $\mathrm{CD}^{+}$or $\mathrm{CD}^{+}$NY-ESO-1-specific T cell clones were then added for overnight culture, after which IFN- $\gamma$ in supernatants was measured. As shown in Figure 4, KBMA $L m N Y$-infected DCs activated both NY-ESO-1-specific CD4 ${ }^{+}$and $\mathrm{CD}^{+} \mathrm{T}$ cell clones. While $\mathrm{CD}^{+} \mathrm{T}$ cell responses were comparable among the 6 donors tested, $\mathrm{CD}^{+} \mathrm{T}$ cell responses varied in magnitude in a donor-specific manner. However, all observed responses were NY-ESO-1-specific and statistically significant when compared with responses elicited by empty vector (Figure 4 and Supplemental Figure 3A). Interestingly, DCs infected with KBMA L. monocytogenes elicited a more pronounced response from the NY-ESO-1-specific $\mathrm{CD}^{+} \mathrm{T}$ cell clones than the DCs infected with live L. monocytogenes when both strains were used at the highest MOI, which does not yet to a large extent impair DC viability (MOI 100 vs. MOI 10). To confirm that presentation of NY-ESO-1 epitopes was not limited to in vitro generated DCs but would take place also when heterogenous APC populations are targeted, PBMCs or monocytes were isolated from blood, infected with $L m N Y$, and used as stimulators for NY-ESO-1-specific T cell clones. These infected APCs were DR or DQ restricted and were cocultured with HLA matched clones overnight when we evaluated NY-ESO-1-specific clonal responses by measurement of IFN- $\gamma$ in culture supernatants (Supplemental Figure 3, B and C). We confirmed that in addition to moDCs, heterogenous PBMC populations and monocytes isolated from blood efficiently processed and presented the full-length NY-ESO-1 protein encoded by L. monocytogenes.

In summary, our data demonstrate that both CD8- and CD4restricted epitopes are efficiently processed and presented to $\mathrm{T}$ cell clones by APCs when the full-length protein is delivered by the L. monocytogenes vector.

KBMA L. monocytogenes-infected DCs prime melanoma antigen-specific effector $C D 8^{+} T$ cells. To be effective in vivo, KBMA L. monocytogenes vectors must confer antigen-specific $T$ cell priming activity to DCs. Therefore, naive $\mathrm{T}$ cells from a healthy individual were cocultured with autologous DCs after infection with KBMA LmAg encoding the Mart-1 epitope. Every 9 days, the T cells were restimulated with KBMA LmAg-infected DCs for a total of 3 stimulations. As shown in Figure 5A, DCs infected with KBMA $L m$ Ag expanded $\mathrm{MP}_{58-66^{-}}$-specific naive $\mathrm{CD}^{+} \mathrm{T}$ cells. Before the onset of priming, no staining of naive $\mathrm{T}$ 

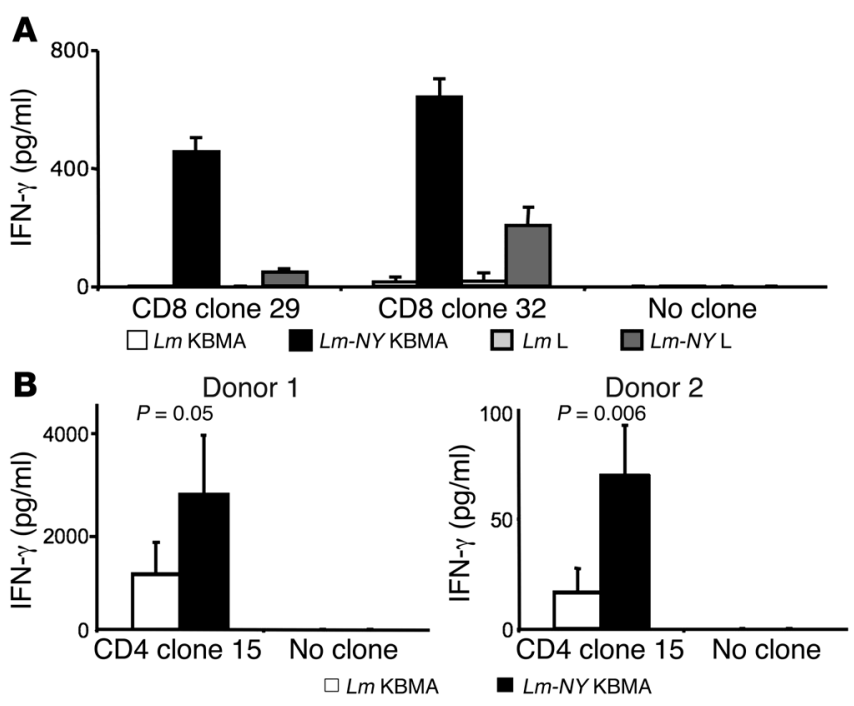

cells was apparent with visualization of tetrameric complexes of HLA-

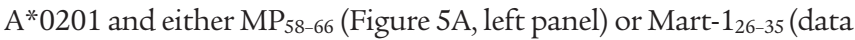
not shown), verifying that the starting $\mathrm{T}$ cell population did not contain significant numbers of MelanA/Mart-1- or MP-specific cells.

We next evaluated whether live $L m A g$ was more efficient at priming naive T cells to Mart- $1_{26-35}$ than KBMA LmAg in our in vitro system. DCs infected with live LmAg (MOI 10) or KBMA LmAg (MOI 50) were used to stimulate $T$ cells as described above, and priming efficacy was tested with Mart- $1_{26-35}$ tetramers. A higher MOI was used for KBMA $L m A g$ to compensate for this strain's inability to replicate inside the infected cell. Both strains were able to prime Mart-126-35-specific T cells at similar frequencies (Figure 5B, 4 left panels). The Mart- $1_{26-35}$ population from the culture stimulated by KBMA $L m A g$-infected DCs was enriched by using a limiting dilution assay of pooled wells in which Mart- $1_{26-35}$ tetramer-positive cells were detected (Figure 5B, 2 right panels). Specific antigen responses were measured by intracellular cytokine secretion. Only Mart- $1_{26-35}$ tetramer-positive cells responded to stimulation with Mart- $1_{26-35}$ peptide-pulsed T2 cells but not to HLA-A*0201-restricted HIV Gag $77-85$ peptide-pulsed T2 cells that we used as a negative control (Figure 5C). In 2 different donors, most of the responding Mart- $1_{26-35}$ tetramer-positive cells produced several cytokines, including IFN- $\gamma$, TNF- $\alpha$, and IL-2 (24\% and $17 \%$ of Mart- $1_{26-35}$ tetramer-positive cells produced all 3 cytokines in both donors) (Figure 5D). Cytotoxicity of the Mart- $1_{26-35}$-specific T cells primed with KBMA LmAg was tested by using a chromium release assay. These cells lysed the MelanA/Mart-1 expressing A2.1 $1^{+}$cell line Gmel but not the A2.1-888 melanoma line (Figure 5E). The lysis of Gmel was blocked when cells were pretreated with an anti-A2.1 antibody. Our data show that $\mathrm{CD}^{+} \mathrm{T}$ cells primed by KBMA $\mathrm{LmAg}$-infected DCs recognize native Mart-1 peptide and are able to execute their effector functions to destroy a melanoma tumor cell line.

In a murine therapeutic tumor model, KBMA L. monocytogenes are more efficient as a vaccine iftargeting APCs in vivo. In terms of targeting DCs for therapeutic treatment of tumors, there are 2 main strategies that can be employed. DCs can be matured and loaded with antigens ex vivo and then injected back into patients. This approach is well established, although DC-based immune therapies remain to be fully optimized. The second approach is to target DCs in vivo, either directly or via intermediate vector "carrier cells" that

\section{Figure 4}

LmNY deliver antigens for presentation by MHC class I and class II molecules. Following infection with NY-ESO-1 protein encoding $\triangle$ inlB $\triangle$ actA $\triangle$ uvrAB L. monocytogenes ( $L m N Y$ ) or the empty vector control (L. monocytogenes) DCs were cocultured with either CD4+ or CD8 ${ }^{+}$NY-ESO-1-specific T cell clones for 18 hours. Supernatants were collected and IFN- $\gamma$ was measured in an ELISA assay. Background IFN- $\gamma$ secretion was determined by DC-only culture. Error bars represent SD of quadruplicate wells of DCs or T cell-DC culture. (A) HLA A2.1+ DCs were infected and cocultured with 2 separate HLA A2.1-restricted NY-ESO-1-specific CD8 ${ }^{+} \mathrm{T}$ cell clones. MOI for infection was 10 for live and 100 for KBMA L. monocytogenes. The experiment is a representative of 6 different donors tested. (B) DCs derived from 2 HLA DR B4*0101 restricted donors (shown are Donors 1 and 2 out of 3 donors tested) were infected with KBMA L. monocytogenes at MOI 100 and cocultured with NY-ESO-1-specific HLA DR B4*0101restricted CD4+ clone 15.

are cross-presented by DCs. Apart from being simpler and cheaper, this approach offers the advantage of targeting multiple DC types in addition to other APCs. Using L. monocytogenes as a vaccine vector makes the second approach even more appealing, since upon i.v. injection, $L$. monocytogenes spontaneously localize in $\mathrm{T}$ cell priming areas of the spleen (26) and deliver antigens for priming by resident DCs (12). It was shown previously that targeting APCs in vivo with KBMA L. monocytogenes encoding tumor antigen mediates priming to tumor antigens, breaks tolerance to the endogenous antigens, and controls tumor growth (11). We have now used a therapeutic vaccination model in CT-26 tumor-bearing mice. We have compared in vivo targeting to ex vivo loading of DCs using KBMA L. monocytogenes expressing the immunodominant $\mathrm{T}$ cell epitope AH1/A5. While ex vivo loading of DCs with KBMA L. monocytogenes-expressing AH1/A5 elicited a small AH1/A5-specific T cell response, the protective effect of the primed cells was insignificant (Figure 6). Both, DCs loaded with vaccine or empty vector mediated similar transient control of tumor growth compared with the untreated mice, likely dependent on stimulation of innate immunity. On the contrary, therapeutic immunization of mice with either live or KBMA recombinant $L$. monocytogenes induced protective immune responses against the tumor that expresses endogenous antigen (Figure 6B) and significantly prolonged survival when mice were followed for 100 days. In summary, KBMA L. monocytogenes expressing tumor antigen is a potent vaccine vector that primes protective $\mathrm{T}$ cell responses in mice, when its recombinant antigen is delivered to the in vivo resident APCs.

\section{Discussion}

Current therapeutic strategies to treat metastatic cancer have either shown limited success in tumor regression or were associated with high toxicities. Therefore, alternative approaches are urgently needed. DCs are attractive adjuvants in humans to increase host resistance to tumors. DCs acquire antigens through several mechanisms, efficiently process and present antigens to $\mathrm{T}$ cells (8), and have an excellent safety record in humans. However, new strategies are needed to optimize their immunogenicity, as there have been few reports demonstrating their ability to induce sustained, protective antitumor immunity in humans $(27,28)$. One such strategy is to use a system that efficiently targets DCs in vivo. In addition to possibly targeting more relevant in vivo resident DC subsets as well as other APCs, such strategies would also considerably lower the cost of the vaccine. 
research article

A

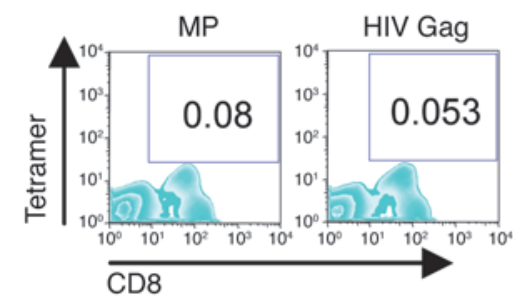

в
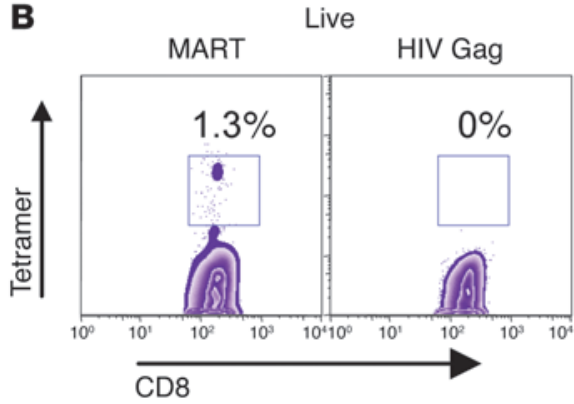

C
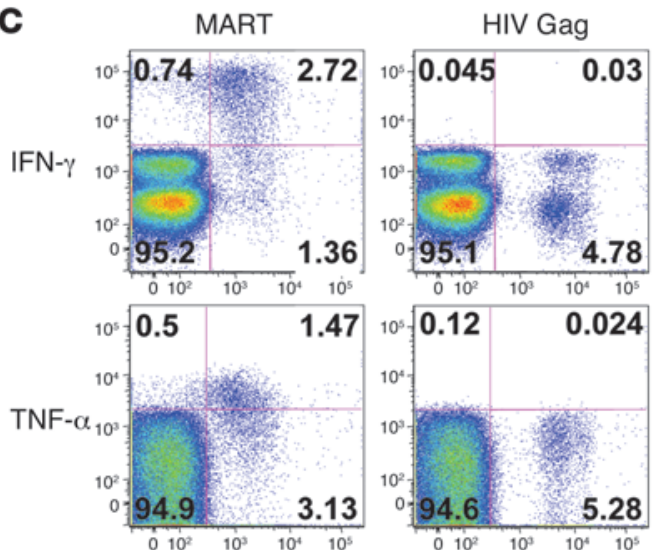

$10^{2}$

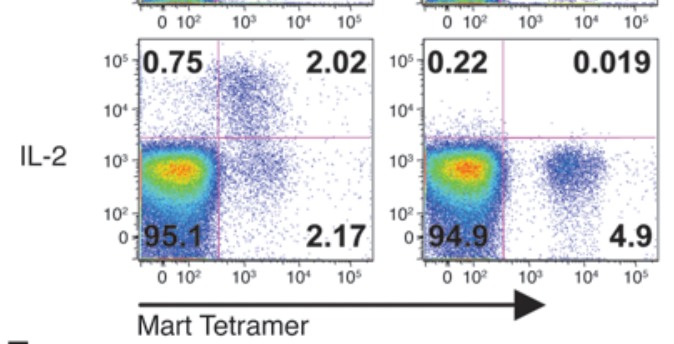

E

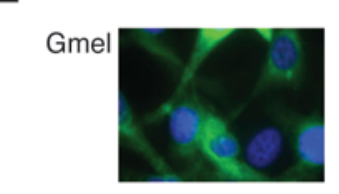

888

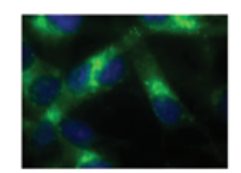

MART
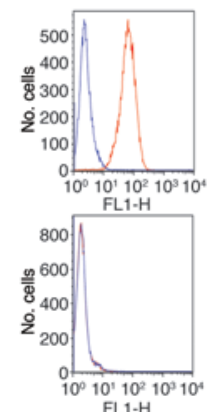

A2.1

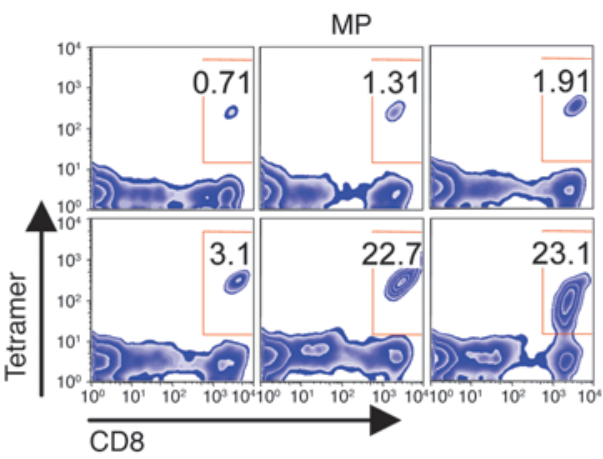

KBMA HIV Gag

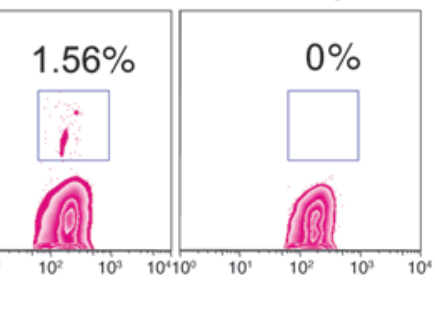

D

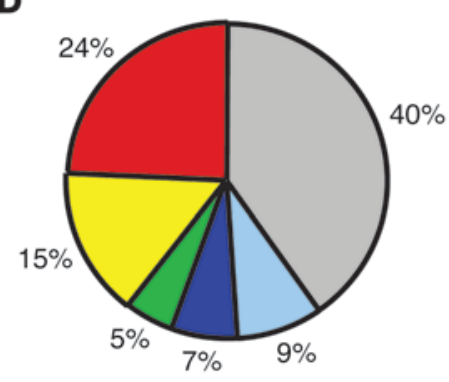

Donor 1
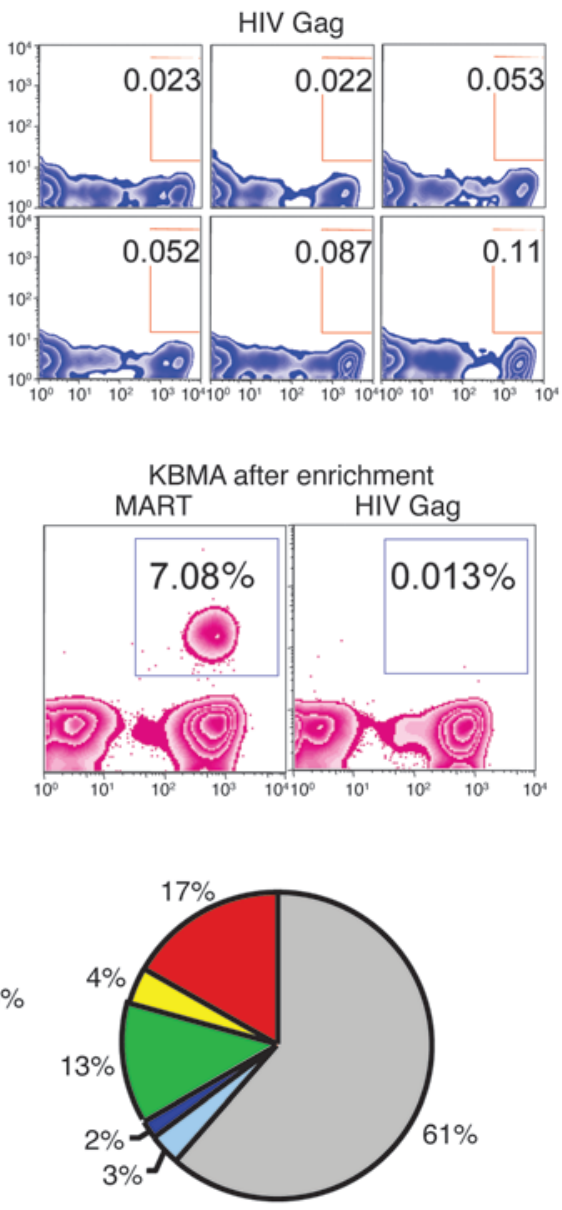

Donor 2
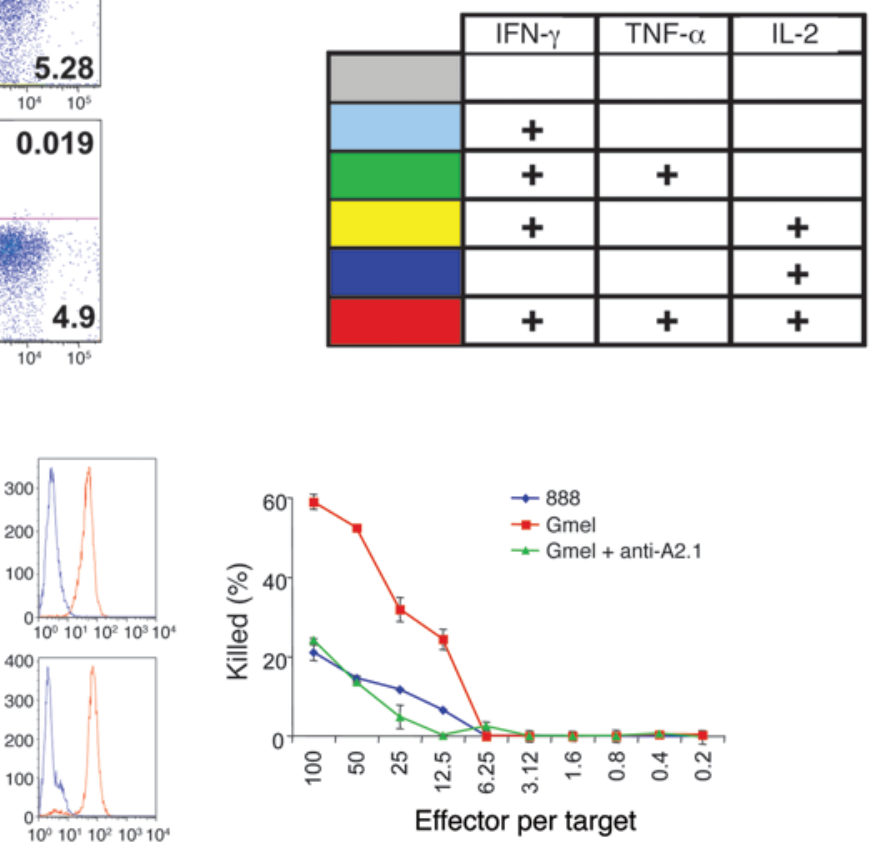

Effector per target 


\section{Figure 5}

Recombinant $L$. monocytogenes prime effector $C D 8^{+} \mathrm{T}$ cells to the encoded recombinant antigens. HLA $A^{*} 0201^{+}$DCs were infected with the KBMA $\triangle a c t A \Delta i n I B \Delta u v r A B$ L. monocytogenes strain expressing the HLA A*0201-restricted CD8+ ${ }^{*}$ cell epitopes of MP and MelanA/ Mart-1. (A) The naive T cell population lacks the MP-tetramer-positive cells (left panels). After stimulation, MP-specific CD8 ${ }^{+} \mathrm{T}$ cells were generated at various frequencies (each middle panel representing a single well). Lack of staining with HIV Gag tetramers assured specificity (right panels). The numbers indicate the percentage of tetramerpositive cells. (B) Both live and KBMA recombinant $L$. monocytogenes primed naive Mart- $1_{26-35}-$ specific T cells at similar frequencies. Percentage of CD3-gated, CD8, and tetramer double-positive cells after priming (left panels) or after the enrichment with limiting dilution assay is shown (right panels). HIV Gag tetramers were used as a negative control. The population of cells enriched for Mart-1-tetramer-positive cells was used for the functional assays shown in $\mathbf{C}-\mathbf{E}$. (C and D) T cells were stimulated with $\mathrm{T} 2$ cells pulsed with Mart- $1_{26-35}$ peptide, and Mart-1-tetramer-positive cells were analyzed for secreted cytokines by intracellular staining. HIV Gag-tetramers were used as specificity controls. In $\mathbf{C}$, the dot plots of 1 , and in $\mathbf{D}$, pie charts representing the spectrum of secreted cytokines by 2 tested donors are shown. In $\mathbf{C}$, the numbers indicate the percentage of cells in each dot plot quadrant. (E) The same $T$ cell populations were tested for cytotoxicity of melanoma cell lines. Gmel and 888 melanoma cell lines express MelanA/Mart-1 protein (left panel), but only Gmel is HLA A*0201-restricted (middle panels), which correlates with the latter's ability to be lysed by MelanA/ Mart-1-specific T cells (right panel). Original magnification, $\times 100$. Preincubation of Gmel with anti-HLA A*0201 antibodies inhibited lysis. A representative result for 1 of 2 donors is shown in $\mathbf{A}-\mathbf{C}$ and $\mathbf{E}$. In $\mathbf{E}$, error bars represent SD of triplicate culture wells.

L. monocytogenes are one of the recombinant microbial vector platforms that has proved to elicit protective responses in a number of experimental models, including infectious and malignant diseases (29-31). L. monocytogenes localize to T cell zones of spleen white pulp and efficiently targets DCs in vivo (26). Development of $\mathrm{T}$ cell anti-L. monocytogenes immunity depends on the presence of CD $11 c^{+}$cells such as DCs $(12,26)$. Next to its remarkable immunogenicity and cost-effectiveness, several other features make L. monocytogenes an intriguing tumor vaccine candidate. These include the ability to break tolerance to self antigens $(11,32,33)$ and the preserved efficacy of the recombinant $L$. monocytogenes vaccines despite preexisting immunity to the bacteria (34).

While only mild toxicity was observed when partially attenuated L. monocytogenes were administered to healthy human volunteers orally (35), it is not considered safe to administer a virulent form of a human pathogen to immunocompromised cancer patients. Efforts to reduce virulence of $L$. monocytogenes have focused on determinants that enable intracytoplasmic escape (LLO), replication, and intercellular spread without exposure to hostile extracellular defense components (ActA and InlB). Among the virulence determinants, LLO is likely one of the major factors in pathogenicity of $L$. monocytogenes; however, its deletion is also associated with a decrease in protectiveness of the $\mathrm{T}$ cells generated in infected mice (36).

Thus, to address the concerns inherent to delivering potentially toxic bacteria to humans, we constructed highly attenuated strains, lacking the proteins Act A and InlB, a strategy that reduces toxicity in mice by 1,000 fold (31). We show that $\triangle a c t A \Delta i n l B$ L. monocytogenes can still efficiently enter human DCs through InlB-independent phagocytic mechanisms and preserve DC-stimulatory capacity through their ability to mature these APCs. To ensure the safety of using L. monocytogenes as a vaccine, a further attenuating step was taken by limiting the ability of the bacteria to replicate. While HK L. monocytogenes are safe to use in mice, the primed $\mathrm{CD}^{+} \mathrm{T}$ cells of the memory repertoire do not acquire effector functions and are not protective from subsequent $L$. monocytogenes infection (37). Immunization of mice with a mixture of live and HK L. monocytogenes does not alter the phenotype of the $\mathrm{CD}^{+} \mathrm{T}$ cells primed by the HK $L$. monocytogenes, thus the absence of an inflammatory milieu cannot alone explain this intrinsic difference (26). Several approaches have been utilized to interfere with the replication of L. monocytogenes while preserving $\mathrm{T}$ cell-stimulatory capacity (38, 39). For clinical application, a well-controlled, cost-effective method with consistent inactivation of L. monocytogenes and full DC-stimulatory potential is preferred. We developed our candidate strain on a recently described vaccine platform that simultaneously takes advantage of the potency of live and the safety of killed microbes (KBMA L. monocytogenes) (11). KBMA vaccine strains were constructed by abrogating the capacity for nucleotide excision repair through deletion of the UV light resistance genes $u v r A$ and $u v r B$. The gene deletion of $u v r A B$ renders the bacteria exquisitely sensitive to photochemical inactivation through the combined treatment of S-59 and UVA. Because of their inability to repair the DNA crosslinks, KBMA L. monocytogenes are unable to replicate and are thus functionally noninfectious (11). The very limited number of crosslinks, however, preserves their metabolic activity, ability to exit the phagolysosome, and ability to escape into the cytosol. L. monocytogenes that are inactivated by high doses of S-59, with a correspondingly increased number of cross-links per genome, have abrogated replication and metabolic activity, similar to the recently described L. monocytogenes inactivated by irradiation (38).

In our studies, the L. monocytogenes with preserved metabolic activity supercede their nonmetabolic counterparts in DC-stimulatory potential (Supplemental Figure 1). The potent maturation stimulus provided by metabolically active $L$. monocytogenes to DCs is likely to be multifactorial. L. monocytogenes activate APCs through ligation of TLRs and through cytoplasmic TLR-independent pathways $(17,40-42)$. In addition, the induction of proinflammatory cytokines such as IL-1, TNF, IL-6, and type I IFN, factors known to activate immature DCs, are likely to amplify these effects.

To prove that highly attenuated KBMA could be used to target tumor antigens to DCs, we constructed recombinant $L$. monocytogenes that contain the MelanA/Mart-1 epitope or L. monocytogenes expressing full-length NY-ESO-1 protein under the LLO promoter. Our studies demonstrate that the proteins are efficiently processed within the infected DCs and MelanA/Mart-1 epitopes are expressed in sufficient amounts on the surface of DCs to prime MelanA/Mart-1-specific $\mathrm{CD}^{+} \mathrm{T}$ cells. KBMA recombinant L. monocytogenes-infected DCs induced cytolytic T cells that recognized native antigen expressed by patient-derived melanoma cells and secreted a panel of effector cytokines, including IFN- $\gamma$, TNF- $\alpha$, and IL-2. Along with the HLA-A*0201-restricted MelanA/Mart-1 epitope, the recombinant $L$. monocytogenes also expressed an MP epitope. Supporting previous observations in the murine system (34), MelanA/Mart-1 priming was not hindered due to preexisting immunity to influenza (data not shown).

Unlike most viral vectors, $L$. monocytogenes constructs are not neutralized by preexisting L. monocytogenes-specific immunity. In addition, $L$. monocytogenes are not restricted by the length of heterologous sequence that can be inserted and thus allows the encoding of full-length proteins. This offers 2 advantages: (a) develop- 
A

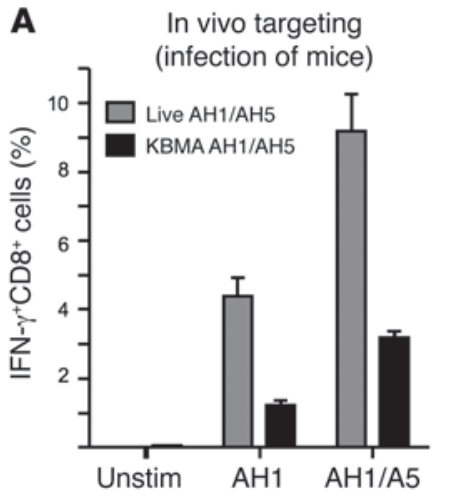

B

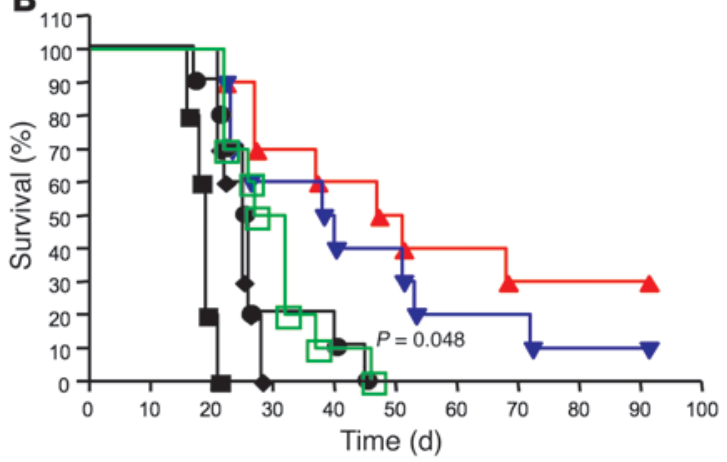

Figure 6

Targeting DCs with KBMA L. monocytogenes-expressing AH1/A5 in vivo controls CT-26 tumor growth. (A) Five mice per group were used for assessment of priming efficacy. The efficacy of $T$ cell priming was compared between mice infected in vivo (left panel) and mice vaccinated with DCs infected in vitro (right panel). Mice were sacrificed 7 days after immunization, and the percentage of antigen-positive IFN- $\gamma$-secreting $\mathrm{CD}^{+} \mathrm{T}$ cells upon in vitro stimulation with $\mathrm{AH} 1$ or $\mathrm{AH} 1 / \mathrm{A} 5$ peptides in the presence of brefeldin A was analyzed by flow cytometry. Error bars represent SD of triplicate culture wells. (B) BALB/c mice were inoculated with CT-26 tumors and immunized 5 days later. They were randomized (10 per group) and vaccinated either with 1 of the indicated DC vaccines or with the live or KBMA L. monocytogenes-expressing AH1/A5. Survival of the mice was monitored over 100 days. $P$ value of survival difference in mice vaccinated with DCs pulsed ex vivo with $L$. monocytogenes-expressing AH1/A5 versus mice infected with $L$. monocytogenesexpressing $\mathrm{AH} 1 / \mathrm{A} 5$ is shown $(P=0.048)$. No significant difference was observed among mice vaccinated with live-attenuated or KBMA $L$. monocytogenes $(P=0.328)$.

ment of a vaccine or immune therapeutic that circumvents the problems of HLA restriction and (b) simultaneous priming of $\mathrm{CD}^{+}$and $\mathrm{CD}^{+} \mathrm{T}$ cells. The second is especially advantageous in tumor settings where $\mathrm{CD}^{+} \mathrm{T}$ cell licensing is necessary for longterm protective $\mathrm{CD}^{+} \mathrm{T}$ cell responses (43-45). We show that L. monocytogenes expressing full-length NY-ESO-1 protein efficiently delivered antigens for both MHC class I and class II presentation across different $\mathrm{MHC}$ restrictions.

While ex vivo maturation and antigen loading of DCs is a wellestablished vaccine approach, which offers the advantage to monitor for vaccine quality before injection into patients, it has yet to prove its efficacy in patients with larger tumor burdens where tolerance to tumor antigens has been established. While prophylactic vaccination in an OVA-expressing tumor model by ex vivo loaded DCs mediated protective $\mathrm{T}$ cell responses to tumor (data not shown), we found that ex vivo loaded DCs had reduced ability to induce protective responses in a therapeutic vaccination model, using CT-26 tumor-bearing mice. In these experiments, initial immunization was also followed by 2 booster vaccinations.
In contrast, targeting DCs in vivo by infecting mice with attenuated KBMA recombinant $L$. monocytogenes primed effector $T$ cells that increased survival of tumor-bearing mice, an effect that was comparable to protection mediated by live L. monocytogenes. While there may be several reasons for the observed differences, the animal data indicate that vaccination strategies may need to be approached differently in tumor-free patients with high likelihood of recurrence versus patients with metastatic disease or unresectable tumors.

In summary, an ideal cancer vaccine would simultaneously induce $\mathrm{CD} 8^{+}$ and $\mathrm{CD}^{+} \mathrm{T}$ cells. This can be made possible by using a vector that encodes a full-length cancer-relevant protein and preferentially targets APCs in vivo. Altogether, our studies show that KBMA $\triangle a c t A \Delta i n l B \Delta$ wr $A B$ recombinant $L$. monocytogenes are a safe, efficient, and cost-effective method of antigen loading and activation/maturation of human DCs with great promise for successful clinical use.

\section{Methods}

Bacterial strains. The L. monocytogenes strains used in this study were derived from the WT L. monocytogenes strain DP-L4056 (46). L. monocytogenes strains with deletion of virulence proteins and $u v r A B$ were prepared as described previously $(11,31)$. The pPL2 integration vector was used to construct recombinant $L$. monocytogenes encoding a single copy of OVA containing the HLA-A*0201restricted $T$ cell epitopes MelanA/Mart- $1_{26-35}$ (ELA modified) and Flu MP $\mathrm{MP}_{5-66}$ and the $\mathrm{H}-\mathrm{K}^{\mathrm{d}}$ restricted AH1/A5 mouse T cell epitope or full-length NY-ESO-1 protein integrated adjacent to the $t R N A^{\text {Arg }}$ gene in the bacterial genome (11). The epitope-containing OVA construct is under the transcriptional control of the bly promoter and is secreted from the bacterium as a fusion protein to the amino terminal 62 aa of LLO. The MelanA/Mart- $1_{26-35}$ and Flu MP S8-66 $_{\text {epitopes were codon-optimized }}$ for L. monocytogenes, synthesized as complementary oligonucleotides with appropriate $5^{\prime}$ and $3^{\prime}$ overhangs, and added to the PPL2-LLO-OVA fusion construct by oligo ligation to unique AvaII and SapI restriction sites within the OVA DNA sequence, respectively. For studies using viable L. monocytogenes, bacteria were grown at $37^{\circ} \mathrm{C}$ in brain-heart-infusion broth to log phase, washed once with PBS, and resuspended in $8 \% \mathrm{DMSO} /$ PBS. Aliquots were frozen and stored at $-80^{\circ} \mathrm{C}$.

S-59/UVA inactivation of bacteria and metabolic activity. L. monocytogenes were grown in $50 \mathrm{ml}$ of brain-heart-infusion broth at $37^{\circ} \mathrm{C}$, with shaking to an $\mathrm{OD}_{600}$ of 0.5 , and S-59 was added directly to the cultures for 1 additional hour. The bacterial cultures were transferred to culture plates and UVA irradiated at a dose of $6.5 \mathrm{~J} / \mathrm{cm}^{2}$ (FX1019 irradiation device; Baxter Fenwal). Photochemically treated bacteria were washed once with PBS and resuspended in $8 \%$ DMSO and stored at $-80^{\circ} \mathrm{C}$ or used directly for experiments. 
Media. RPMI-1640 (Cellgro) supplemented with 1 mM HEPES (Gibco) and $1 \%$ plasma (Valley Biomedical) was used for infection and culture of human DCs with $L$. monocytogenes. For ELISPOT assay and mixed lymphocyte reaction, media were supplemented with $20 \mu \mathrm{g} / \mathrm{ml}$ gentamicin (Gibco) and 5\% pooled human serum (PHS; ValleyBiomedical) instead of $1 \%$ plasma. For maintenance of lines and for the Chromium-51 (Cr-51) release assay, cells were cultured in RPMI-1640 supplemented with $1 \mathrm{mM}$ HEPES and 10\% FBS (Gibco). Yssel's T cell medium (Gemini Bio-Products) supplemented with 5\% PHS was used in the T cell priming assays. For priming of NY-ESO-1-specific T cells, we used complete IMDM media (Gibco) (IMDM with 5\% PHS, glutamine, and nonessential amino acids; Sigma-Aldrich) supplemented with 100 units $/ \mathrm{ml}$ penicillin, $0.1 \mathrm{mg} / \mathrm{ml}$ streptomycin, $150 \mathrm{IU} / \mathrm{ml} \mathrm{IL-2} \mathrm{(R \& D} \mathrm{Systems),} \mathrm{and} 10 \mathrm{ng} / \mathrm{ml}$ of IL-7 (R\&D Systems). For NY-ESO-1-specific T cell-DC cocultures, complete IMDM supplemented with $20 \mathrm{IU} / \mathrm{ml} \mathrm{IL-2}$ and gentamicin was used.

Peptides. H-2K ${ }^{\mathrm{d}}$-restricted epitope AH1 or the altered AH1/A5 (SPSYAYHQF) were synthesized commercially (SynPep) and used as previously

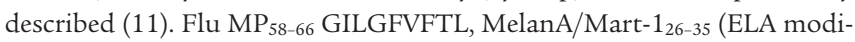
fied) ELAGIGILTV, and HIV Gag ${ }_{77-85}$ SLYNTVATL peptides were synthesized by Genemed Synthesis Inc. A library of overlapping peptides spanning the full length of the NYESO protein was obtained from ProImmune Inc.

Generation of $\mathrm{CD} 4^{+}$and $\mathrm{CD} 8^{+} \mathrm{NY}$-ESO-1 $\mathrm{T}$ cell clones. $\mathrm{CD} 4^{+} \mathrm{NY}$-ESO-1-specific clones were generated as described previously (47). Briefly, PBMCs were stimulated repetitively with the pool of overlapping peptides spanning the complete NY-ESO-1 aa sequence (P119-143, P139-160, the remaining peptides were 20 mers overlapping by 10 aa). After 3 restimulations, antigen-specific $\mathrm{T}$ cells were enriched by IFN- $\gamma$ capture assay (Miltenyi Biotec). The cells were then cloned by limiting dilution assay. We seeded 0.3 cells/well of 96 -well plate and expanded cells polyclonally with PHA-L (Sigma-Aldrich). NY-ESO-1specific cells were determined by measurement of intracellular IFN- $\gamma$ upon stimulation with each of the NY-ESO-1 peptides in the presence of brefeldin A (Sigma-Aldrich). CD8 ${ }^{+}$NY-ESO- 1 cells were obtained from a patient that was vaccinated with NY-ESO-1 amino acids 157-165 (SLLMWITQV), with a $\mathrm{V}$ substituted for the naturally occurring $\mathrm{C}$ at position 165 for better HLA-A2 binding. NY-ESO-1-specific CD8 ${ }^{+} \mathrm{T}$ cells were expanded by stimulation with NY-ESO $157-165$ peptide in the presence of $1,000 \mathrm{IU} / \mathrm{ml} \mathrm{IL-2}$ for 10 days as previously published (48). APCs were then cloned and tested as described above,

Human PBMCs, DCs, APCs, and naive T cells. moDCs were generated from buffy coats (New York Blood Center) or leukaphereses (BRT Laboratories Inc.) as described previously (49); however, unless noted, addition of gentamicin to the media was omitted. Monocytes were obtained from buffy coats by $\mathrm{CD} 14^{+}$magnetic selection (Miltenyi Biotech), and blood DCs were obtained by FACS sorting as described (18). T cells for priming were obtained from buffy coats, leukapheresis, or patient blood. Buffy coats were purchases from New York Blood Center, leukophereses were purchased from BRT Laboratories Inc., and usage of patients' blood was approved by New York University Institutional Review Board.

Infection of DCs with L. monocytogenes and immunofluorescence staining. Immature human DCs were seeded to 24-well plates at $10^{6}$ cells/well or a 96-well plate at $1.5 \times 10^{5}$ cells/well and were incubated with $L$. monocytogenes for 1 hour at $37^{\circ} \mathrm{C}$. Adherent cells were washed twice with RPMI containing $50 \mu \mathrm{g} / \mathrm{ml}$ gentamicin (Gibco) and kept in medium supplemented with $20 \mu \mathrm{g} / \mathrm{ml}$ gentamicin. In NY-ESO-1 experiments, DCs were infected in rotating tubes for 1 hour, washed, counted, and cocultured with $\mathrm{T}$ cells in U-bottom plates (Costar).

Human DCs were infected at an MOI of 1 or 10 for viable bacteria or an MOI of 20, 50, 100, or 200 for KBMA L. monocytogenes or $\Delta$ bly L. monocytogenes for 60 minutes at $37^{\circ} \mathrm{C}$. Extracellular bacteria were removed by washing. For fluorescence microscopy, infected cells were incubated for 5 hours at $37^{\circ} \mathrm{C}$ on poly-L-lysine-coated coverslips in the presence of gentamicin, then fixed with $3.5 \%$ formaldehyde. Following permeabilization with TBS Triton X-100, DCs on coverslips were stained with rabbit anti-L. monocytogenes antibody (BD Diagnostics), followed by FITC-conjugated goat anti-rabbit (Vector Laboratories). Polymerized actin was visualized using phalloidinrhodamine (red) (Molecular Probes) and coverslips were mounted using ProLong Gold antifade with DAPI (blue) (Molecular Probes). For detection of MelanA/Mart-1 expression in melanoma cell lines, adherent cells grown on chamber slides (BD) were fixed and stained intracellularly with Mart-1specific antibodies (Novacastra) and FITC-conjugated goat anti-mouse secondary antibody (Jackson ImmunoResearch Laboratories Inc.).

Assessment of human DC maturation. Following infection with L. monocytogenes, the DCs were cultured for 36 hours further unless otherwise noted. They were then stained with antibodies against CD80, CD83, CD86, HLA-A, -B, -C (all from BD Biosciences), or CCR7 (R\&D Systems). The expression of the maturation markers was quantified according to MFI or percentage of positively stained cells on a FACSCalibur (BD Biosciences - Pharmingen).

Measurement of cytokines. DCs cultured with various stimuli were incubated for 36 hours at $37^{\circ} \mathrm{C}$ before their culture supernatants were harvested and tested for the presence of the following cytokines: IL-6, IL-1 $\beta$ (both from R\&D Systems), IL-12p70, TNF- $\alpha$ (both from BD Biosciences - Pharmingen), and IFN- $\alpha$ (PBL) and chemokines MCP-3 and RANTES (both from R\&D Systems) by ELISA. IFN- $\gamma$ (Biosource) was similarly measured in supernatants of T cell-DC cocultures. Alternatively, in selected experiments, cytokines produced by DCs were measured by FACS using human inflammatory Cytometric Bead Array (BD).

Antigen presentation. DCs were infected with either live (MOI 10) or KBMA (MOI 50 or MOI 100) L. monocytogenes (both LmAg expressing MP and Mart epitopes and $L$. monocytogenes without antigen) in a 24-well plate as described above and cultured for 36 hours at $37^{\circ} \mathrm{C}$. The cells were harvested, counted, and cocultured with $\mathrm{MP}_{58-66}$-specific T cell clones (50) in an IFN- $\gamma$ capture ELISPOT plate (Millipore) for at least 16 hours. Subsequently, the plate was developed according the manufacturer's protocol, and IFN- $\gamma$ spots were quantified with an automated counter (AID). For the antigen presentation kinetics assay, DCs were plated in the wells of a 96-well plate and infected with L. monocytogenes as described above. At 6, 9, and 12 hours after infection, the cells were fixed as described, and Flu-specific $\mathrm{T}$ cells were added directly to the wells containing the fixed DCs for 16 hours at which point the supernatants were harvested for an IFN- $\gamma$ ELISA. In NY-ESO- 1 experiments, DCs were infected for 1 hour in a rotating tube, treated with gentamicin, and put on ice for 30 minutes. $\mathrm{CD}^{+}$or $\mathrm{CD}^{+} \mathrm{T}$ cell clones were then added to DCs in U-bottom plates at a DC/T cell ratio of 1:3 for 18 hours at which point supernatants were collected and IFN- $\gamma$ was measured by ELISA.

Cell fixation. In the antigen presentation kinetics assay, cells were fixed with $2 \%$ PFA (Sigma-Aldrich) for 10 minutes, washed with DMEM, and incubated with L-lysine for 20 minutes to neutralize the effects of PFA. L-lysine (Sigma-Aldrich) was prepared by diluting $36.54 \mathrm{~g}$ of L-lysine in $500 \mathrm{ml}$ distilled water.

Priming. DCs infected with $L$. monocytogenes were cocultured with naive syngenic $\mathrm{T}$ cells derived from PBMCs by magnetic depletion (Miltenyi Biotech) of CD19-, CD14-, CD56-, and CD45RO-positive cells in the presence of IL-6 $(1,000 \mathrm{IU} / \mathrm{ml})(\mathrm{R} \& D$ Systems $)$ and IL-12 $(5 \mathrm{ng} / \mathrm{ml})$. The T cells were restimulated every 9 days with the $L$. monocytogenes-infected DCs in the presence of IL-2 (10 U/ml) (Chiron) and IL-7 (5 ng/ml) (R\&D Systems). A week after the third stimulation, the cells were analyzed for the presence of Mart $26-35$ tetramer-positive cells. Antigen-specific $\mathrm{T}$ cells were then enriched by limiting dilution assay as described above with 10 cells seeded per well. Wells that contained more than $5 \%$ of specific cells were pooled and frozen in aliquots for further testing. For allogeneic priming (mixed lymphocyte reaction), PBMCs were depleted of CD19-, CD14-, CD56-, CD8-, and CD45RO-positive cells by magnetic beads (Miltenyi Biotech) and cocultured with allogeneic DCs. 
Cell lines and blocking of HLA 2.1. Melanoma patient-derived cell lines, Gmel and 888, were used to test HLA-A2-restricted CTL function. Both express MelanA/Mart-1, but only Gmel is HLA-A2 positive, therefore 888 was used as a negative control. The $\mathrm{T} 2$ cells deficient in transporter associated protein were pulsed with peptides and used to stimulate HLA-A2restricted $\mathrm{CD}^{+} \mathrm{T}$ cells in the intracellular cytokine staining assay. Antibodies against human HLA A2.1 (clone BB7-2, HB82; ATCC) were used to show HLA-restriction of the CTL responses.

Generation of tetramers. PE-labeled tetrameric MHC-peptide complexes $\left(\mathrm{MP}_{58-66} /\right.$ HLA-A*0201, MelanA/Mart-1 ${ }_{26-35} /$ HLA-A*0201, and HIV gag ${ }_{77-85}$ / HLA-A*0201) were synthesized by the Vaccine and Cell Therapy Facility at New York University Medical Center as described previously (51). Plasmids encoding human $\beta_{2}$ microglobulin and HLA-A2 were a gift from Christian Munz of The Rockefeller University.

Tetramer and intracellular cytokine staining. T2 cells were pulsed with Mart $26-35$ peptides or HIV Gag $77-85$ peptides (both at $1 \mu \mathrm{g} / \mathrm{ml}$ ) for 2 hours at $4{ }^{\circ} \mathrm{C}$ in RPMI-1640. After extensive washing the peptide-pulsed $\mathrm{T} 2$ cells were cocultured with polyclonal $\mathrm{T}$ cells containing Mart-specific cells in the presence of $10 \mu \mathrm{g} / \mathrm{ml}$ of brefeldin A (Sigma-Aldrich) for 6 hours at $37^{\circ} \mathrm{C}$. After the incubation, the cells were stained with a PE-conjugated tetramer of Mart $26-35$ /HLA-A*0201 complexes, CD4 Alexa Fluor 700, CD8 PerCP-Cy5.5 and CD3 APC-Cy7 antibodies (BD Biosciences - Pharmingen). Next, the cells were fixed and stained intracellularly as previously described (52) with FITCconjugated IFN- $\gamma$, PE-Cy7-conjugated TNF, and IL-2-APC antibodies (BD Biosciences - Pharmingen) and analyzed with an LSR flow cytometer.

Chromium release assay. Melanoma cell lines were labeled with $\mathrm{Cr}-51$ (PerkinElmer) at a final concentration of $1 \mathrm{mCi} / \mathrm{ml}$ for 90 minutes at $37^{\circ} \mathrm{C}$. After washing twice with RPMI-1640 supplemented with $10 \%$ FBS, they were cocultured with Mart-specific polyclonal $T$ cells at various ratios for 6 hours. Cr-51 released into the supernatants was measured with a Wallac 1450 Microbeta counter (PerkinElmer). The percentage of cells killed was calculated by dividing specific release from maximum release, after first subtracting spontaneous release from both samples.

Mice and immunizations. Six- to eight-week-old female BALB/c (Charles River Laboratories) were handled according to US National Institutes of Health guidelines. All protocols requiring animal experimentation received prior approval from the Anza Therapeutics Animal Care and Use Committee. All vaccinations for immunogenicity and tumor studies were by i.v. injection using either $1 \times 10^{8} \mathrm{KBMA}$ or $1 \times 10^{6}$ live attenuated L. monocytogenes or $1-2 \times 10^{6} \mathrm{DCs}$. Initial immunization was followed by 2 booster immunizations 14 days apart.

Generation and suspension of murine DCs. DCs were generated as described previously (53). Briefly, BM from tibias and femurs of BALB $/ \mathrm{c}$ mice was cultured in RPMI-1640 plus 10\% FBS and GM-CSF $(20 \mathrm{ng} / \mathrm{ml}$ murine GM-CSF; R\&D Systems). Ten days later, nonadherent cells were harvested and verified phenotypically to be myeloid DCs (MHC class II hi CD11 $\mathrm{c}^{\text {hi }}$,

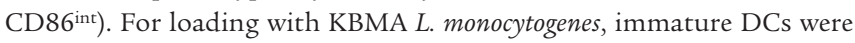
incubated in suspension with KBMA L. monocytogenes for 1 hour at MOI of 20. Extracellular bacteria were washed away and cells placed back into suspension culture at $37^{\circ} \mathrm{C}$. Fifteen hours later, cells were washed 3 times and resuspended in $\mathrm{dPBS}$ for injection.
Tumor studies. We i.v. implanted BALB/c mice with $2 \times 10^{5} \mathrm{CT}-26$ cells. We randomized mice 3 days later into experimental groups, 10 animals per group, and vaccinated them. Mice were injected with HBSS and vaccinated with 1 of the DC vaccines (DCs and DCs infected with KBMA $\Delta a c t \Delta i n l \Delta u v r A B$ L. monocytogenes-expressing AH1/A5 or its empty KBMA vector control) or were inoculated i.v. with live or KBMA $\triangle a c t \Delta i n l \Delta u v r A B$ L. monocytogenes-expressing AH1/A5 bacteria. AH1/A5 is the heteroclitic epitope of the AH1 epitope of tumor antigen gp70, naturally presented by the CT-26 tumor. Survival of the mice was monitored over 100 days. Mice were sacrificed if they started to show any signs of stress or labored breathing. Satellite mice ( 5 per group) were used for assessment of priming efficacy. Mice were sacrificed 7 days after immunization and the percentage of antigen-positive IFN- $\gamma$-secreting $\mathrm{CD}^{+} \mathrm{T}$ cells, upon in vitro stimulation with AH1 or AH1/A5 peptides in the presence of brefeldin A, was analyzed by flow cytometry.

Statistics. Statistical significance among DCs infected by different $L$. monocytogenes strains were tested by 2 -tailed Student's $t$ test. $P$ values of less than 0.05 were considered significant. Mean values of 5 mice were calculated to enumerate the responses to $\mathrm{AH} 1$ or $\mathrm{AH} 1 / \mathrm{A} 5$ peptides. Tumor survival results were analyzed by Prism "Survival curve" statistical analysis.

\section{Acknowledgments}

Supported by grants from the National Cancer Institute (CA84512), the National Institutes of Health (AI044628, AI061684, AI057127), the Bill \& Melinda Gates Foundation, the Mary Kirkland Foundation, and the Cancer Research Institute. N. Bhardwaj is a Doris Duke Distinguished Scientist and an Elizabeth Glaser Scientist. We thank David O'Neill and Sylvia Adams for providing melanoma patient PBMCs. The tetramers were prepared by the New York University Cancer Institute Vaccine and Cell Therapy Core Facility.

Received for publication December 21, 2006, and accepted in revised form October 1, 2008.

Address correspondence to: Mojca Skoberne or Nina Bhardwaj, New York University School of Medicine, 522 First Avenue, SML 1307, New York, New York 10016, USA. Fax: (212) 263-6729; E-mail: Mojcaskoberne@yahoo.de (M. Skoberne). Phone: (212) 263-5814; Fax: (212) 263-6729; E-mail: Nina.Bhardwaj@nyumc.org (N. Bhardwaj).

Keith S. Bahjat's present address is: Earle A. Chiles Research Institute, Robert W. Franz Cancer Research Center, Providence Portland Medical Center, Portland, Oregon, USA.

Mojca Skoberne's present address is: Genocea Biosciences, Cambridge, Massachusetts, USA.

Mojca Skoberne, Alice Yewdall, and Keith S. Bahjat contributed equally to this work.
1. Ribas, A., Butterfield, L.H., Glaspy, J.A., and Economou, J.S. 2003. Current developments in cancer vaccines and cellular immunotherapy. J. Clin. Oncol. 21:2415-2432.

2. Speiser, D.E., et al. 2005. Rapid and strong human CD8+ T cell responses to vaccination with peptide, IFA, and CPG oligodeoxynucleotide 7909. J. Clin. Invest. 115:739-746.

3. Powell, D.J., Jr., and Rosenberg, S.A. 2004. Phenotypic and functional maturation of tumor antigen-reactive CD8+ T lymphocytes in patients undergoing multiple course peptide vaccination. J. Immunother. 27:36-47.

4. Rosenberg, S.A., et al. 2005. Tumor progression can occur despite the induction of very high levels of self/tumor antigen-specific CD8+ T cells in patients with melanoma. J. Immunol. 175:6169-6176.

5. Dudley, M.E., et al. 2005. Adoptive cell transfer therapy following non-myeloablative but lymphodepleting chemotherapy for the treatment of patients with refractory metastatic melanoma. J. Clin. Oncol. 23:2346-2357.

6. Dranoff, G. 2005. CTLA-4 blockade: unveiling immune regulation. J. Clin. Oncol. 23:662-664.

7. Rosenberg, S.A., and Dudley, M.E. 2004. Cancer regression in patients with metastatic melanoma after the transfer of autologous antitumor lymphocytes. Proc. Natl. Acad. Sci. U. S. A. 101(Suppl. 2):14639-14645.

8. O’Neill, D.W., Adams, S., and Bhardwaj, N. 2004. 
Manipulating dendritic cell biology for the active immunotherapy of cancer. Blood. 104:2235-2246.

9. Skoberne, M., and Geginat, G. 2002. Efficient in vivo presentation of Listeria monocytogenesderived CD4 and CD8 T cell epitopes in the absence of IFN-gamma. J. Immunol. 168:1854-1860.

10. Kolb-Maurer, A., et al. 2000. Listeria monocytogenes-infected human dendritic cells: uptake and host cell response. Infect. Immun. 68:3680-3688.

11. Brockstedt, D.G., et al. 2005. Killed but metabolically active microbes: a new vaccine paradigm for eliciting effector T-cell responses and protective immunity. Nat. Med. 11:853-860.

12. Jung, S., et al. 2002. In vivo depletion of $\mathrm{CD} 11 \mathrm{c}^{+}$ dendritic cells abrogation priming of $\mathrm{CD}^{+} \mathrm{T}$ cells by exogenous cell-associated antigens. Immunity. 17:211-220.

13. Zammit, D.J., Cauley, L.S., Pham, Q.M., and Lefrancois, L. 2005. Dendritic cells maximize the memory CD8 T cell response to infection. Immunity. 22:561-570.

14. Neuenhahn, M., et al. 2006. CD8alpha+ dendritic cells are required for efficient entry of Listeria monocytogenes into the spleen. Immunity. 25:619-630.

15. Brzoza, K.L., Rockel, A.B., and Hiltbold, E.M. 2004. Cytoplasmic entry of Listeria monocytogenes enhances dendritic cell maturation and $\mathrm{T}$ cell differentiation and function. J. Immunol. 173:2641-2651.

16. Feng, H., et al. 2005. Listeria-infected myeloid dendritic cells produce IFN-beta, priming $\mathrm{T}$ cell activation. J. Immunol. 175:421-432.

17. Stockinger, S., et al. 2004. IFN regulatory factor 3 dependent induction of type I IFNs by intracellular bacteria is mediated by a TLR- and Nod2-independent mechanism. J. Immunol. 173:7416-7425

18. Fonteneau, J.F., et al. 2001. Generation of high quantities of viral and tumor-specific human CD4+ and CD8+ T-cell clones using peptide pulsed mature dendritic cells. J. Immunol. Methods. 258:111-126.

19. Mercado, R., et al. 2000. Early programming of T cell populations responding to bacterial infection. J. Immunol. 165:6833-6839.

20. Ayyoub, M., et al. 2006. A phenotype based approach for the immune monitoring of NY-ESO-1specific $\mathrm{CD} 4+\mathrm{T}$ cell responses in cancer patients. Clin. Immunol. 118:188-194.

21. Valmori, D., et al. 2007. Epitope clustering in regions undergoing efficient proteasomal processing defines immunodominant CTL regions of a tumor antigen. Clin. Immunol. 122:163-172.

22. Gnjatic, S., et al. 2006. NY-ESO-1: review of an immunogenic tumor antigen. Adv. Cancer Res. 95:1-30.

23. Barrow, C., et al. 2006. Tumor antigen expression in melanoma varies according to antigen and stage. Clin. Cancer Res. 12:764-771.

24. Velazquez, E.F., et al. 2007. Expression of the cancer/testis antigen NY-ESO- 1 in primary and meta- static malignant melanoma (MM)--correlation with prognostic factors. Cancer Immun. 7:11.

25. Adams, S., et al. 2008. Immunization of malignant melanoma patients with full-length NY-ESO-1 protein using TLR7 agonist imiquimod as vaccine adjuvant. J. Immunol. 181:776-784.

26. Muraille, E., et al. 2005. Distinct in vivo dendritic cell activation by live versus killed Listeria monocytogenes. Eur. J. Immunol. 35:1463-1471.

27. O'Neill, D., and Bhardwaj, N. 2005. Exploiting dendritic cells for active immunotherapy of cancer and chronic infection. Methods Mol. Med. 109:1-18.

28. Dillman, R.O., Selvan, S.R., and Schiltz, P.M. 2006. Patient-specific dendritic-cell vaccines for metastatic melanoma. N. Engl. J. Med. 355:1179-1181.

29. Li, Z., Zhao, X., Higgins, D.E., and Frankel, F.R. 2005. Conditional lethality yields a new vaccine strain of Listeria monocytogenes for the induction of cellmediated immunity. Infect. Immun. 73:5065-5073.

30. Gunn, G.R, et al. 2001. Two Listeria monocytogenes vaccine vectors that express different molecular forms of human papilloma virus-16 (HPV-16) E7 induce qualitatively different $\mathrm{T}$ cell immunity that correlates with their ability to induce regression of established tumors immortalized by HPV-16. J. Immunol. 167:6471-6479.

31. Brockstedt, D.G., et al. 2004. Listeria-based cancervaccines that segregate immunogenicity from toxicity. Proc. Natl. Acad. Sci. U. S. A. 101:13832-13837.

32. Singh, R., Dominiecki, M.E., Jaffee, E.M., and Paterson, Y. 2005. Fusion to Listeriolysin O and delivery by Listeria monocytogenes enhances the immunogenicity of HER-2/neu and reveals subdominant epitopes in the FVB/ $\mathrm{N}$ mouse. J. Immunol. 175:3663-3673.

33. Bruhn, K.W., Craft, N., Nguyen, B.D., Yip, J., and Miller, J.F. 2005. Characterization of anti-self CD8 T-cell responses stimulated by recombinant Listeria monocytogenes expressing the melanoma antigen TRP-2. Vaccine. 23:4263-4272.

34. Starks, H., et al. 2004. Listeria monocytogenes as a vaccine vector: virulence attenuation or existing antivector immunity does not diminish therapeutic efficacy. J. Immunol. 173:420-427.

35. Angelakopoulos, H., et al. 2002. Safety and shedding of an attenuated strain of Listeria monocytogenes with a deletion of act $\mathrm{A} / \mathrm{plcB}$ in adult volun teers: a dose escalation study of oral inoculation. Infect. Immun. 70:3592-3601.

36. Bahjat, K.S., et al. 2006. Cytosolic entry controls CD8+-T-cell potency during bacterial infection. Infect. Immun. 74:6387-6397.

37. Lauvau, G., et al. 2001. Priming of memory but not effector CD8 T cells by a killed bacterial vaccine. Science. 294:1735-1739.

38. Datta, S.K., et al. 2006. Vaccination with irradiated Listeria induces protective $\mathrm{T}$ cell immunity. Immunity. 25:143-152.

39. Schoen, C., et al. 2005. Bacterial delivery of func- tional messenger RNA to mammalian cells. Cell. Microbiol. 7:709-724.

40. Edelson, B.T., and Unanue, E.R. 2002. MyD88dependent but Toll-like receptor 2 -independent innate immunity to Listeria: no role for either in macrophage listericidal activity. J. Immunol. 169:3869-3875.

41. Torres, D., et al. 2004. Toll-like receptor 2 is required for optimal control of Listeria monocytogenes infection. Infect. Immun. 72:2131-2139.

42. O'Connell, R.M., et al. 2005. Immune activation of type I IFNs by Listeria monocytogenes occurs independently of TLR4, TLR2, and receptor interacting protein 2 but involves TNFR-associated NF kappa B kinase-binding kinase 1. J. Immunol. 174:1602-1607.

43. Janssen, E.M., et al. 2003. CD4+ T cells are required for secondary expansion and memory in CD8+ T lymphocytes. Nature. 421:852-856

44. Shedlock, D.J., and Shen, H. 2003. Requirement for CD4 T cell help in generating functional CD8 T cell memory. Science. 300:337-339.

45. Williams, M.A., Tyznik, A.J., and Bevan, M.J. 2006. Interleukin-2 signals during priming are required for secondary expansion of CD8+ memory T cells. Nature. 441:890-893.

46. Lauer, P., Chow, M.Y., Loessner, M.J., Portnoy, D.A., and Calendar, R. 2002. Construction, characterization, and use of two Listeria monocytogenes site-specific phage integration vectors. J. Bacteriol. 184:4177-4186.

47. Godefroy, E., et al. 2006. Identification of two Melan-A CD4+ T cell epitopes presented by frequently expressed MHC class II alleles. Clin. Immunol. 121:54-62.

48. Montes, M., et al. 2005. Optimum in vitro expansion of human antigen-specific CD8 T cells for adoptive transfer therapy. Clin. Exp. Immunol. 142:292-302.

49. Lee, A.W., et al. 2002. A clinical grade cocktail of cytokines and PGE(2) results in uniform maturation of human monocyte-derived dendritic cells: implications for immunotherapy. Vaccine. 20(Suppl. 4):A8-A22.

50. Fonteneau, J.F., et al. 2001. Generation of high quantities of viral and tumor-specific human CD4+ and CD8+ T-cell clones using peptide pulsed mature dendritic cells. J. Immunol. Methods. 258:111-126.

51. Altman, J.D., et al. 1996. Phenotypic analysis of antigen-specific T lymphocytes. Science. 274:94-96.

52. Fonteneau, J.F., et al. 2003. Activation of influenza virus-specific CD4+ and CD8+ T cells: a new role for plasmacytoid dendritic cells in adaptive immunity. Blood. 101:3520-3526.

53. Lutz, M.B., et al. 1999. An advanced culture method for generating large quantities of highly pure dendritic cells from mouse bone marrow. J. Immunol. Methods. 223:77-92. 